\section{Estrategias para el fortalecimiento de emprendimientos informales}

\section{Strategies for the strengthening of informal enterprises}

\section{Ximena Cervantes Molina* \\ Guadalupe Murillo Campuzano* \\ Francisco Liberio Roca*}

\section{RESUMEN}

La presente investigación estuvo dirigido a los emprendimientos informales dirigidos por mujeres en el cantón Quevedo, Provincia de Los Ríos. El fin fue establecer las determinantes que influyen para que una mujer participe laboralmente y se concentre en el comercio informal y establecer estrategias para mejorar sus negocios. Se aplicó estadística descriptiva. EI universo estuvo conformado por el total de mujeres que se dedican al comercio informal en el cantón Quevedo; se aplicó un muestro probabilístico aleatorio a 136 negocios informales; se analizaron variables aleatorias continuas y discretas. Se determinó que la media en la edad de las mujeres que se dedican al comercio informal es de $4 \mathrm{I}$ años con un mínimo de 15 años y un máximo de 80 años; el $44,60 \%$ de mujeres se dedica a la venta de ropa siendo la actividad más representativa y en menor orden se encuentran emprendimientos de comida y calzado en un $16,11 \%$ y $11,37 \%$ en su orden. Así mismo se conoció que en un $90 \%$ las emprendedoras no realizan planes estratégicos para posicionarse en el mercado. Se concluye que es importante implementar estrategias competitivas en emprendimientos informales ya que crea el potencial de rendimiento que necesitan para obtener beneficios en el futuro.

\footnotetext{
* Master, Universidad Técnica Estatal de Quevedo, Quevedo Ecuador, xcervantes@uteq.edu.ec, http://orcid.org/0000-0002-2300-4288

* Master, Universidad Técnica Estatal de Quevedo, Quevedo Ecuador, gmurillo@uteq.edu.ec, http://orcid.org/0000-0003-2362-0723

* Master, Universidad Técnica Estatal de Quevedo, Quevedo Ecuador, fliberio@uteq.edu.ec, https://orcid.org/0000-0002-3043-4032
}

\section{REVISTATECNOLÓGICA ciencia y educación Edwards Deming}

ISSN: 2600-5867

Atribución/Reconocimiento-NoCo mercial- Compartirlgual 4.0 Licencia Pública Internacional - CC

\section{BY-NC-SA 4.0}

https://creativecommons.org/licenses /by-nc- sa/4.0/legalcode.es

Editado por: Tecnológico Superior Corporativo Edwards Deming Julio - Diciembre Vol. 5 - 2 - 202 I https://revista-edwardsdeming.com/index.php/es e-ISSN: 2576-097।

Recibido: 27 junio 2020

Aprobado: 09 Enero, 202।

Pag $117-130$ 
Palabras clave: FODA, economía informal, estrategias, equidad de género

\begin{abstract}
The present investigation was directed to the informal enterprises directed by women in the Quevedo canton, Los Ríos Province. The purpose was to establish the determinants that influence a woman to participate in the labor market and focus on informal commerce and to establish strategies to improve her businesses. Descriptive statistics were applied. The universe was made up of the total number of women who are dedicated to informal commerce in the Quevedo canton; a random probability sample was applied to 136 informal businesses; Continuous and discrete random variables were analyzed. It was determined that the average age of women engaged in informal trade is $4 \mathrm{I}$ years with a minimum of 15 years and a maximum of 80 years; $44.60 \%$ of women are dedicated to the sale of clothing, being the most representative activity and in less order are food and footwear enterprises in $16.11 \%$ and $11.37 \%$ in their order. Likewise, it was known that $90 \%$ of the entrepreneurs do not carry out strategic plans to position themselves in the market. It is concluded that it is important to implement competitive strategies in informal enterprises since it creates the performance potential they need to obtain benefits in the future.
\end{abstract}

Keywords: SWOT, informal economy, strategies, gender equity

\title{
INTRODUCCIÓN
}

La informalidad laboral es uno de los mayores desafíos de los países en situación de desarrollo; esta problemática involucra temas que van desde los riesgos a los que se exponen los trabajadores para conseguir un ingreso mínimo, hasta las elevadas presiones sobre los sistemas de seguridad social debido a los miles de individuos que no aportan a salud y pensión. (Castiblanco, 2018). La relación entre informalidad y género no es casual o irrelevante. De hecho, es la expresión de las condiciones desiguales en las que las mujeres entran al trabajo remunerado. La desigualdad horizontal en el retorno de la educación y la mayor flexibilidad de estos empleos, que permite compatibilizar mejor el empleo y las tareas reproductivas a su cargo, son solo dos de los factores que explican la alta participación de mujeres en empleos informales (Arango y Pineda, 2012; Espino, 20I2).

Las mujeres aún participan en desventaja frente a los hombres: por cada hora trabajada, las mujeres reciben ingresos laborales que son, en promedio, $17 \%$ inferiores a los de los hombres con la misma edad, educación, presencia de niños en sus hogares, presencia de otros generadores de ingresos en el hogar, condición de ruralidad y tipo de trabajo. Cuando la comparación de salarios de hombres y mujeres incluye además la dedicación laboral semanal de los trabajadores (es decir, el número de horas trabajadas por semana), la brecha alcanza el $27 \%$. Las condiciones desfavorables de las mujeres en los 
mercados de trabajo no se restringen a los salarios: la participación laboral femenina alcanza poco más de dos tercios de la masculina, la tasa de desempleo femenina excede a la masculina y persisten las barreras culturales en el ingreso de hombres y mujeres a segmentos importantes de los mercados de trabajo predominantemente femeninos (servicio doméstico) y predominantemente masculinos (minería) (OIT, 2019).

El Panorama Laboral de América Latina y el Caribe 2019 da cuenta de un momento de incertidumbre para mujeres $y$ hombres que forman parte de la población económicamente activa y sus familias. La lectura de los indicadores de los mercados de trabajo no es positiva, pues aumenta la desocupación y hay señales de menor calidad en los empleos.

Un estudio del Banco Interamericano de Desarrollo (BID) titulado 'Participación Laboral Femenina', ubica a Ecuador en el último lugar de toda Sudamérica, en el mercado laboral. Mientras en países como Uruguay o Perú, 8 de cada 10 mujeres dentro de la Población Económicamente Activa (PEA) tienen un empleo. En el país solo el 65,6\% de ese grupo poblacional tiene participación en el mercado laboral. Una cifra que apenas ha mejorado en un $2 \%$ en los últimos cuatro años, del $63,6 \%$ al $65,6 \%$. Además, solo el $29,8 \%$ de ellas tiene un empleo adecuado (INEC, 2019).

En el caso de Ecuador, la tasa de desocupación en el año 2019 alcanza el 4,6\% superior al 4,2\% del año 20।8; además la tasa de desocupación de las mujeres alcanza el I,3\%, lo que quiere decir que existe una brecha alta con relación a los varones. Igualmente, existe un incremento del trabajo por cuenta propia en un 2,2\%, el servicio doméstico el $1,2 \%$ con relación al trabajo asalariado entre el año 2018 y el 2019.

Asimismo, según la Encuesta Nacional de Empleo, Desempleo y Subempleo (ENEMDU) (Instituto Nacional de Estadísticas y Censos [INEC], 2019), en Ecuador el sector formal representa el $47 \%$; el sector informal el $46,5 \%$, y el empleo doméstico $2,3 \%$; también, la encuesta muestra que el empleo adecuado representa el 19,5\% y de desempleo el 2,2\%, delos cuales el $0,3 \%$ son ocultos y el I,9\% son abiertos. En Ecuador el comercio es una actividad formal, que representa el $32,2 \%$ superior al $26,8 \%$ con relación a América Latina, siendo la mayor actividad desarrollada por la población económicamente activa de género femenino, que representa el $42,4 \%$ con respecto a los varones que alcanza el $25 \%$, seguido por las actividades relacionada con los servicios, Sin embargo, el comercio es desarrollado no solo como una actividad formal, sino también informal, considerándose como un síntoma a nivel mundial, y un fenómeno complejo y de múltiples dimensiones que comprende varios aspectos como: Sociales, económicos, históricos y formativos.

En la Provincia de Los Ríos la población considera al comercio informal como una alternativa de fuente de ingresos y como una forma de satisfacer sus necesidades propias de las cuales esta categorizado como subempleo y corresponde a un 53,10\%. Las personas acceden a este tipo de economía por diversos motivos, entre ellos, la búsqueda de una mejor calidad de vida para ellos y sus familias, las facilidades de entrada (ya que el capital inicial es bajo y asequible para cualquier persona), los rápidos ingresos que 
reciben en corto tiempo y en algunos casos porque es su única oportunidad para satisfacer sus necesidades básicas. La presente investigación busca establecer las determinantes que influyen para que una mujer participe laboralmente y se concentre en el comercio informal y establecer estrategias para sus negocios.

Los aspectos teóricos que respaldan y se revisan en este estudio son los relacionados con los diferentes conceptos acerca del emprendimiento y sus características generales, así como las distintas connotaciones teóricas y prácticas atribuidas a la informalidad, las condiciones más importantes asociadas a la creación de empresas en el aspecto formal, la repercusión que las diversas formas de actividad económica, formal e informal, tienen sobre el crecimiento económico y su potencial desarrollo posterior (Véliz, et al., 20l4). La palabra "emprendimiento" guarda estrecha relación con el vocablo francés entrepreneur, que aparece a inicios del siglo XVI para describir al sujeto "que se encarga de una obra". Ravier (2009) se remite al trabajo de Richard Cantillón quien, en I750, definió el término como "la voluntad o capacidad de enfrentar la incertidumbre", incorporando con ello el factor riesgo; es decir que el emprendedor o empresario es aquella persona que emprende una actividad productiva bajo su cuenta y riesgo. El aspecto riesgo tiene como eje central la posibilidad de pérdida del capital invertido; lo inverso sería la posibilidad de que la actividad desarrollada tuviera éxito y se alcanzaran beneficios financieros y económicos. Dicho concepto de emprendedor perduró hasta comienzos del siglo XX. No obstante, Schumpeter (1934) introdujo una noción fundamental para explicar el desarrollo de los negocios; esta se refiere al fenómeno denominado "destrucción creativa", que sugiere que el proceso de innovación constituye la clave del crecimiento económico. Lógicamente, se espera que quienes implementen ese cambio de manera práctica sean los emprendedores. El concepto de "destrucción creativa" involucra conceptos adicionales como "costo de oportunidad", que no es otra cosa que el costo de dejar de hacer una actividad para realizar otra; evidentemente, la realización de la otra actividad el hecho de que la misma proporcionará un rendimiento superior al obtenido con anterioridad. En consecuencia, la "destrucción creativa" lleva implícito el dejar de hacer una tarea para efectuar otra mediante un proceso de innovación que permitirá aumentar la producción y el rendimiento económico financiero. Posteriormente, Kirzner (1973), en su trabajo sobre el empresario, establece que este toma decisiones en función de su conocimiento acerca de las posibles ganancias por obtener, sin negar el hecho de que el empresario no conoce completamente la evolución de un mercado, tan solo intuye la posibilidad de ganancias potenciales. El trabajo de De Soto (2009) desarrolla de forma temprana una serie de condicionantes que influyen sobre el comportamiento del emprendedor, desde el punto de vista de la imposibilidad de afianzar su emprendimiento debido a las múltiples trabas de tipo institucional (tiempo, reglas y pasos a cumplir, realidad impositiva, entre otras), que derivan en un emprendimiento de tipo informal. A finales del siglo pasado se difundieron definiciones e interpretaciones acerca del trabajo informal, todas con un enfoque en común: una explicación legal y una explicación económica. La primera 
caracteriza la informalidad como una actividad ilegal y marginal que no genera impuestos y la segunda la explica como la situación de incapacidad de generar suficientes plazas formales de empleo (Campos, 2008). En la actualidad, a esta concepción se le presta especial atención en el debate sobre el bienestar en relación con la seguridad social y programas de lucha contra la pobreza. Un componente ampliamente aceptado para explicar la informalidad es que la mayor integración económica de los mercados no posibilita, tanto al sector público como al privado, generar suficientes empleos (Koujianou y Pavcnik, 2003; Galiani y Sanguinetti, 2003). Aunque la informalidad se considere un sector residual en términos de contribución a la economía, se ha mostrado evidencia de que el crecimiento de la informalidad no es del todo despreciable para el Estado, puesto que ha sido capaz de disimular problemas estructurales de la economía y generar empleo (Schneider y Klinglmair, 2004; Charmes, 2000).

Por otra parte el emprendimiento femenino contribuye, por una parte, a impulsar el crecimiento económico a partir de la creación de empresas y, por otra, a reforzar las políticas de igualdad. La importancia adquirida por esta actividad se pone de manifiesto en las investigaciones sobre el tema, centradas en analizar los factores que condicionan el emprendimiento por razón de género $y$, especialmente, las razones que explican la menor participación de la mujer en este proceso (Olaz et al., 2017).

Gran parte de la literatura sobre el tema en cuestión ha puesto de manifiesto que, a pesar de las iniciativas institucionales orientadas a la puesta en marcha de la actividad emprendedora femenina ésta, sin embargo, se mantiene en unas tasas de participación inferiores a las de los hombres. La cautela y prevención de la mujer - posiblemente como resultado de ciertas construcciones sociales - ayuda a explicar, el menos en parte, esta realidad.

Relación entre la estrategia y el emprendimiento empresarial De acuerdo con Valencia, et al., 2017), el emprendimiento empresarial y la gestión estratégica son procesos dinámicos que se ocupan del comportamiento y el rendimiento de las empresas. Mientras que la gestión estratégica exige a las empresas establecer y explotar las ventajas competitivas dentro de un medio en particular, el emprendimiento promueve la búsqueda de ventajas competitivas a través de innovaciones de producto, de proceso y de mercado. Los autores han determinado que las acciones emprendedoras $y$ estratégicas tienen por finalidad encontrar un nuevo mercado o espacio competitivo en el que la empresa pueda incursionar para crear riqueza. Fundamentalmente, las empresas tratan de encontrar nuevas formas de hacer negocios que van a alterar las reglas de competencia existentes en una industria, lo que lleva al desarrollo de nuevos modelos de negocio que crean formas diferenciales de vida competitiva (Hitt \& Ireland, 2000).

El término de sector informal fue utilizado por vez primera hace ya más de 35 años por la Organización Internacional del Trabajo (OIT) en un informe sobre Kenia, que a su vez hacía referencia a un artículo de Hart de 1970. La novedad del concepto consistía en 
que trataba de recalcar el hecho de que en los países en desarrollo el problema del empleo se centra más que en el desempleo abierto, en un fenómeno distinto: el conformado por un amplio grupo de trabajadores, que estando ocupados, laboran con un nivel muy bajo de productividad e ingresos insuficientes. Se trata de los llamados "trabajadores pobres" que laboran en unidades productivas de pequeño tamaño, con uso de tecnologías simples, escasos requerimientos de capital por persona ocupada y una escasa división entre la propiedad de los medios de producción y los recursos para el sustento familiar. Prolifera el trabajo familiar y por cuenta propia, junto a la mano de obra asalariada sin prestaciones ni contrato escrito. Al pasar del tiempo se encontró que el término "sector informal" resultaba inadecuado, o engañoso, para poder reflejar los aspectos dinámicos, heterogéneos y complejos de un fenómeno, que en realidad no se concentraba en un solo sector, o grupo industrial de actividad. En su lugar, la OIT propuso el término de "economía informal", que hace referencia a un grupo cada vez más diverso y creciente de trabajadores y empresas que tanto en las áreas urbanas como en las rurales operan de manera informal. Incluyen a trabajadores por cuenta propia en actividades de supervivencia, tales como vendedores ambulantes, boleros, cuidadores de automóviles; empleados domésticos, personas que laboran en su propio domicilio o en talleres, como trabajadores asalariados, como aprendices, o como familiares sin remuneración (Samaniego, 2008).

El sector informal puede describirse en términos generales como un conjunto de unidades dedicadas a la producción de bienes o la prestación de servicios con la finalidad primordial de crear empleos y generar ingresos para las personas que participan en esa actividad. Estas unidades funcionan típicamente en pequeña escala, con una organización rudimentaria, en la que hay muy poca o ninguna distinción entre el trabajo y el capital como factores de producción. Las relaciones de empleo -en los casos en que existanse basan más bien en el empleo ocasional, el parentesco o las relaciones personales y sociales, y no en acuerdos contractuales que supongan garantías formales; las personas ocupadas en estas unidades de producción de bienes o prestaciones de servicios son consideradas dentro del empleo informal. (Ramos, 2015).

Indicadores de medición de la informalidad Las empresas se clasifican de acuerdo a su actividad económica, y las del sector informal por los trabajadores que laboran por cuenta propia o por tener empleadores informales. Asimismo, se toma en cuenta el lugar de trabajo (si es fijo o no), su situación geográfica (rural-urbana), el número de personas ocupadas, tipo de propiedad (propiedad individual, propiedad del hogar, en asociación con miembros de otro hogar), las relaciones con otras empresas (productores independientes, o que trabajan con otras empresas) (OIT, 20I3). El tipo de información que se debe recabar para el sector informal de acuerdo a las recomendaciones de la $15^{\mathrm{a}}$. CIET, es la siguiente: características sociodemográficas (sexo, edad, educación, condiciones laborales: horas de trabajo, tipo de contrato, lugar de trabajo, ingresos). Según Trejo (2004), el marco metodológico para estimar la ocupación en el sector informal a través de encuestas de empleo se hace mediante una estimación y 
caracterización de la ocupación en este sector, siendo las variables seleccionadas: rama de actividad, tamaño de la empresa, nombre del negocio o de la empresa, lugar de trabajo, posición en el trabajo, tipo de contrato, tipo de prestaciones sociales y tipo de empresa.

La población ocupada en este sector comprende: trabajadores por cuenta propia en actividades económicas manufactureras de hasta quince trabajadores y en actividades no manufactureras de hasta cinco trabajadores; empleadores en actividades económicas manufactureras de hasta quince trabajadores y no manufactureras de hasta cinco trabajadores cuyas empresas funcionan sin nombre o registro, y su actividad la realizan en un local $\circ$ en pequeños locales o talleres; trabajadores sin pago en actividades manufactureras de hasta quince trabajadores y no manufactureras de hasta cinco trabajadores cuyas empresas funcionan sin nombre $\circ$ registro, en actividades manufacturas de hasta quince trabajadores y no manufactureras de hasta cinco trabajadores y cuyas empresas funcionan con algún nombre o registro y su actividad la realizan sin un local $\circ$ en pequeños locales 0 talleres; trabajadores asalariados comprende a los que trabajan con contrato de hasta dos meses, a los trabajadores asalariados con contrato de dos a seis meses, trabajadores asalariados sin contrato escrito y sin prestaciones sociales que laboran en actividades manufactureras de hasta quince trabajadores y no manufactureras de hasta cinco trabajadores cuyas empresas funcionan sin nombre o registro, en actividades económicas manufactureras de hasta quince trabajadores y no manufactureras de hasta cinco trabajadores y cuyas empresas funcionan con algún nombre o registro y su actividad la realizan sin un local o en pequeños locales o talleres. Para Trejo (2004), el sector informal o el no estructurado lo comprenden la población ocupada que labora en micro negocios no agropecuarios asociados a los hogares, excepto en servicios domésticos, que no tienen registro ante las autoridades y ocupados que aun cuando laboran en micro negocios establecidos y registrados carecen de un contrato y seguridad social.

Ramos Soto (2007), menciona que los patrones y los trabajadores que laboran por su cuenta forman parte del sector informal; así mismo, señala que están incluidos la economía subterránea, las actividades ilegales como la piratería, la producción y tráfico de drogas, la usura, reventa de taquillas, los vendedores ambulantes de mercancías y de alimentos, artesanos, transportistas, prestadores de servicio doméstico de todo tipo, así como sexo servidoras. Por lo que para este sector crea un índice en el que incorpora indicadores tales como: población ocupada que no tiene servicios médicos, población ocupada que no percibe salario, población que trabaja con familiares sin recibir ingreso alguno, y trabajadores por cuenta propia. El objetivo de este índice es englobar los diferentes conceptos que mencionan la Organización Internacional del Trabajo (OIT) y el Programa Regional del Empleo para América Latina y el Caribe (PREALC). 
Según Saldarriaga et al., 2018; los niveles de informalidad aumentan a medida de que la empresa es más pequeña; estas reflejan su baja capacidad para llevar a cabo actividades rentables y con potencial de expansión que les permitan cubrir los costos de operar en el sector formal de la economía; según el DNP (2007), su acceso a la tecnología es restringido por lo que se pueden ver pequeñas empresas con materiales y maquinaria atrasadas y casi obsoletas, así como personas no calificadas e inexpertas en todo el tema de ventas, preparación de sus productos y sin un mayor conocimiento del servicio al cliente. Un porcentaje significativo de estas empresas vende sus productos y servicios en nichos de mercado locales; sus consumidores son, en su mayoría, de bajos ingresos, y los requisitos de calidad, precio y volumen son poco exigentes. Basados en estas descripciones, es posible considerar una relación directa entre la microempresa y la informalidad. No obstante no son iguales, ya que no todas las microempresas nacen en la informalidad y se desarrollan bajo márgenes de ilegalidad. Los países han diseñado soluciones creativas que consisten en incluir una gran parte de personas que poseen estas unidades de negocio en algún tipo de sistema de protección social, buscan reducir los costos de formalización de las empresas, crean entidades de apoyo económico y social e incentivos para quienes generen nuevos empleos, especialmente para los grupos vulnerables, como los trabajadores jóvenes menores de 28 años, dice la OIT (2013).

\section{MATERIALES Y MÉTODOS}

La presente investigación se la desarrolló a través de la estadística descriptiva. El universo estuvo conformado por el total de mujeres que se dedican al comercio informal en el cantón Quevedo; para tal efecto se obtuvo información estadística en el Gobierno Autónomo Descentralizado Municipal del Cantón Quevedo. Se conoció que existen 21 I mujeres registradas en el municipio que se dedican a diferentes actividades económicas en calidad de comercio informal; se aplicó un muestro probabilístico aleatorio simple considerando un $95 \%$ de nivel de confianza y un 5\% de error dando como resultado una muestra de 136 negocios informales. Para garantizar la validez y confiabilidad de la información, se aplicó un muestreo piloto considerando el $10 \%$ de la muestra a fin de aprobar el instrumento de investigación a aplicar mismo que estuvo conformado por variables aleatorias continuas y discretas.

\section{RESULTADOS}

Se conoció que la media en la edad de las mujeres que se dedican al comercio informal es de $4 \mathrm{I}$ años con un mínimo de 15 años y un máximo de 80 años; referente a su estado civil se estableció que el $33,33 \%$ son solteras, $27,27 \%$ se encuentran en unión libre; el $18,94 \%$ son casadas y en menor orden divorciadas, viudas y en unión de hecho. Por otra parte se determinó que el 9I,94 \% tienen hijo en un número promedio por mujer de 3 hijos. Referente al nivel académico se pudo constatar que el $52,55 \%$ de las mujeres terminaron la secundaria y en 32,12 terminó primaria. Así mismo se pudo establecer que en un $44,60 \%$ de mujeres se dedica a la venta de ropa siendo la actividad más 
representativa y en menor orden se encuentran emprendimientos de comida y calzado en un $16, \mathrm{I} 1 \%$ y $\mathrm{II}, 37 \%$ en su orden (Figura I).

Figura I. Tipos de Emprendimientos Informales dirigidos por mujeres en el Cantón Quevedo

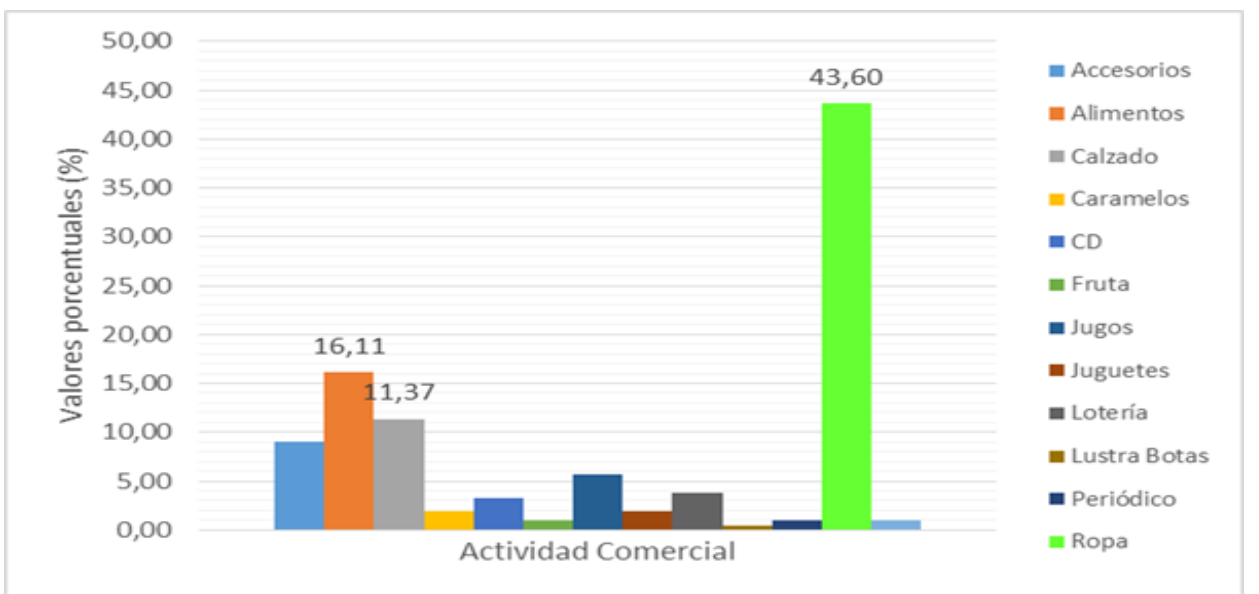

Por otra parte; referente a la cantidad de años que las mujeres llevan dedicadas a sus actividades informales se determinó una media de 19 años con un mínimo de 1 y un máximo de 58 años (Figura 2).

Figura 2. Años que llevan dedicados los emprendimientos Informales dirigidos por mujeres en el Cantón Quevedo

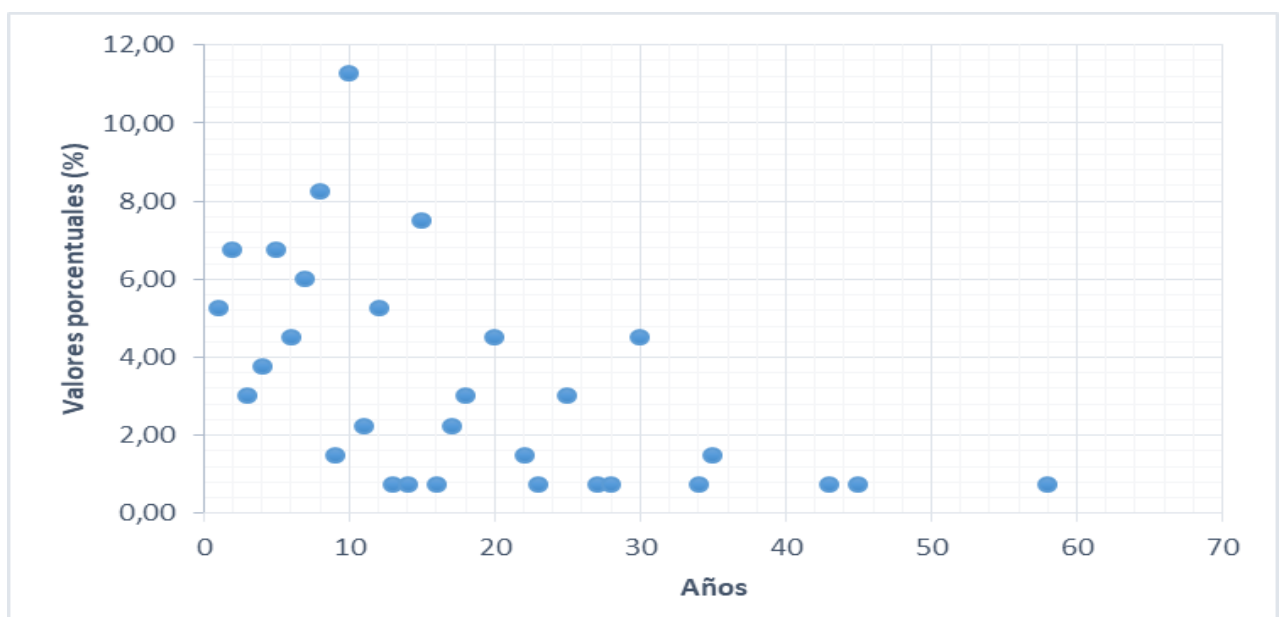

Se analizó que el motivo que direccionó a que las mujeres se dedicarán a ser comerciantes informales fue por razones económicas en un 77,94\%; así mismo se estableció que el $88 \%$ de los negocios informales no tiene RUC mismo que es un 
instrumento que tiene por función registrar e identificar a los contribuyentes con fines impositivos y proporcionar esta información a la Administración Tributaria.

Referente a la aplicación del proceso administrativo por parte de las mujeres que se encuentran inmersas en actividades informales se pudo constatar que el $95 \%$ desconoce de técnicas administrativas lo que conlleva a que no contemplan la planificación estratégica para poder dar cumplimiento a los objetivos estratégicos de los emprendimientos; no presentan una organización jerarquizada con su personal, respecto a la dirección el $92,92 \%$ no han sido capacitadas y en cuanto al control se analizó que $67,89 \%$ realiza el registro de sus ingresos y gastos diariamente sin seguir ningún plan de operaciones.

Toda vez que se ha realizado un análisis de la situación de los emprendimientos informales con la participación femenina se procede a realizar un FODA aplicando la matriz específica.

\section{Fortalezas}

$\checkmark$ Años de experiencia en sus actividades

$\checkmark$ Segmentación de mercado definido

$\checkmark$ Horario de trabajo mayor amplio

$\checkmark$ Predisposición del género femenino a asistir a capacitaciones

$\checkmark$ Personal con nivel académico aceptable

\section{Debilidades}

$\checkmark$ Escasa de capacitación en técnicas administrativas y de mercadotecnia

$\checkmark$ Estancamiento empresarial

$\checkmark$ No poseen una estructura organizacional definida

$\checkmark$ Sus altos niveles de informalidad

$\checkmark$ Sus bajos niveles de asociactividad

$\checkmark$ La estrechez de los mercados a los que dirigen sus productos

$\checkmark$ El bajo nivel tecnológico y de formación de sus recursos humanos

$\checkmark$ El limitado acceso al sector financiero.

$\checkmark$ Carencia en el uso de marketing para obtener una ventaja competitiva

$\checkmark$ Incumplimiento al Fisco

\section{Amenazas}

$\checkmark$ Barreras de entrada

$\checkmark$ Economía cambiante

$\checkmark$ Ingreso de competidores

$\checkmark$ Requisitos de capital, para ingresar a nuevos mercados

$\checkmark$ Falta de productividad empresarial

$\checkmark$ Aparición de nuevas tecnologías en relación a los procesos de producción

$\checkmark$ Fuerte competencia

$\checkmark$ Inestabilidad Política 


\section{Oportunidades}

$\checkmark$ Impulso a la innovación productiva a través de la creación de nuevos emprendimientos

$\checkmark$ Convenios por parte de IES para dar capacitaciones

$\checkmark$ Desarrollo de la Agenda 2030

$\checkmark$ Análisis de la igualdad entre los géneros y empoderamiento a todas las mujeres

$\checkmark$ Reformas que otorguen a las mujeres igualdad de derechos a los recursos económicos

Como estrategias generalizadas, que coadyuven al crecimiento y sustentabilidad de los emprendimientos informales se sugieren las siguientes estrategias.

$\checkmark$ Abrir la oportunidad para que estos negocios informales hagan una declaración voluntaria de su existencia y operación de su negocio. En contraprestación, estos negocios no son penalizados, y entran en un proceso de rápida formalización para obtener acceso al apoyo financiero temporal que se brindan a las empresas formales.

$\checkmark$ Definir estrategias en función de los objetivos, dividiéndolas en las 4 P's de Marketing (Producto, Plaza, Precio y Promoción) integrando elementos como el Servicio, Branding, Comunidades, etc. Con esto la empresa romperá los paradigmas del "miedo al marketing" y vera los beneficios de primera mano.

$\checkmark$ Establecer un diseño organizacional estratégico sustentado en la visión, la misión, los objetivos, que se adapten a las necesidades primordiales de la empresa, señalando su ruta empresarial.

$\checkmark$ Establecer objetivos a corto plazo, considerando las metas concretas y cuantificables en periodos pequeños, para implantar las pautas básicas que deberá seguir la empresa en forma consecutiva y cíclica, permitiendo la eficiencia y eficacia de recursos.

$\checkmark$ Analizar la necesidad de educación económica y financiera, junto con un marco legal para su implementación a través de programas que promuevan las dependencias gubernamentales o privadas, con el objetivo de establecer metodologías y lineamientos que garanticen la correcta distribución y coordinación de los recursos y genere mayor rentabilidad económica.

\section{DISCUSIÓN}

El funcionar como negocio informal tiene demasiadas desventajas, ya que en cualquier momento podrían tener algún problema legal, así como su crecimiento y desarrollo 
empresarial se ve limitado y complicado al no poder realizar una comercialización completa al seguir funcionando de esta manera ilegal. (Arango, 20 I I; Little, 2009)

Es pertinente mencionar que el número de hijos es uno de los factores principales en la participación de la población femenina en el sector informal; debido a que la informalidad les brinda un trabajo remunerado con horarios flexibles que le proporcionan tiempo para ser responsables tanto de su trabajo como de su hogar, caso que se observa en los resultados de este estudio, pues sugieren que un mayor número de hijos abarca mayores posibilidades de ser informales.

Los años de educación son relevantes para este estudio; la relación inversa con respecto a la probabilidad de la participación femenina cuencana dentro del mercado informal, indica que la mayoría de las mujeres prefieren capacitarse y con ello poder formar parte del mercado formal sea en el sector público como privado.

Al analizar la participación femenina en el mercado informal por nivel educativo para el año 2014 en la ciudad de Cuenca, existe una mayor proporción de mujeres en niveles de educación media y superior (Ver Anexo 8), lo que permite concluir que mujeres que alcanzan un alto nivel de educación se están privando de acceder al mercado de trabajo a desempeñar actividades fuera de su área de preparación, por lo que deciden permanecer inactivas esperando una mejor oportunidad de empleo; mientras que mujeres con bajos niveles de educación son indiferentes a los tipos de oficios que se les ofertan ya que se rigen por la situación económica de su hogar.

La responsabilidad familiar no remunerada está destinada principalmente hacia las mujeres; la capacidad de las mujeres de pertenecer a un trabajo remunerado en la ciudad y en el Ecuador depende de sus responsabilidades en la materia del cuidado de los hijos así como en actividades del hogar. El ser jefe de familia para una mujer ha sido un determinante relevante en la decisión de ser informal, ya que son las encargadas directas del sustento de la familia.

\section{REFERENCIAS}

Arango, L. G. (20II). El trabajo de cuidado: iservidumbre, profesión o ingeniería emocional? En L. G. Arango y P. Molinier (Eds.), El Trabajo y la Ética del Cuidado (pp. 9I-109). Medellín, Colombia: La Carreta Editores \& Universidad Nacional de Colombia.

Arango, L. G. y Pineda, J. (20I2). Género, trabajo y desigualdades sociales en peluquerías y salones de belleza de Bogotá. Revista CS, (I0), 93-130. http://dx.doi.org/10.18046/recs.il 0.1356 
Castiblanco-Moreno, S. E. Emprendimiento informal y género: una caracterización de los vendedores ambulantes en Bogotá. Sociedad y Economía, (34), 211-228. https://www.doi.org//0.25100/sye.v0i34.6479

Campos, G. (2008). XI Jornadas de Economía Crítica: La economía informal y sus posibilidades de desarrollo. México Ecocri: Universidad Autónoma de Puebla.

Charmes, J. (2000). Cuarto Encuentro Estadístico del Sector Informal: The contribution of informal sector to GDP in developing countries: Assessment, estimates, methods, organizations for the future. Génova: Delhi Group.

De Soto, H. (2009). El otro Sendero: Una respuesta económica a la violencia. Bogotá Colombia: Editorial Norma.

DNP- Departamento Nacional de Planeación (2007). Política nacional para la transformación productiva y la promoción de las micro, pequeñas y medianas empresas: un esfuerzo público-privado. Documento CONPES, Departamento nacional de planeación, República de Colombia, 30p

Espino, A. (2012). Perspectivas teóricas sobre género, trabajo y situación del mercado laboral latinoamericano. En V. Esquivel (Ed.), La economía feminista desde América Latina: Una hoja de ruta sobre los deberes actuales en la región (pp. 190-246). Santo Domingo, República Dominicana: ONU Mujeres.

Espino, A. (2005). Un marco de análisis para el fomento de las políticas de desarrollo productivo con enfoque de género. Serie: mujer y desarrollo, 77. Santiago, Chile: CEPAL.

Finanzas y Ciencias Administrativas, 19 (37), 90-97. [Fecha de Consulta 10 de Marzo de 202I]. ISSN: 2077-I886. Disponible en: https://www.redalyc.org/articulo.oa?id=3607/3607336/8004

Galiani, S. y Sanguinetti, P. (2003). The impact of trade liberalization on wage inequality: evidence from Argentina. Journal of Development Economics, 72/2, 325.

Hitt, M. A. \& Ireland, R. D. (2000). The intersection of entrepreneurship and strategic management research. Handbook of entrepreneurship, 45, 63.

INEC, 2019. Ecuador, a la cola en participación femenina en el mercado laboral. http://www.youthemploymentdecade.org/es/repor/espanol-ecuador-a-la-cola-enparticipacion-femenina-en-el-mercado-laboral/

Kirzner, I. (1973). Competition and entrepreneurship. Chicago, Estados Unidos: University of Chicago Press

Koujianou, G. y Pavcnik, N. (2003). The response of the informal sector to trade liberalization. Journal of Development Economics, 72(2), 463.

OIT, O. I. (20I3). La medición de la Informalidad: Manual estadístico sobre el sector informal y el empleo informal. Turín, Italia: Centro Internacional de Formación de la OIT.

OIT - Organización Internacional del Trabajo (2005). Economía Informal en las Américas: situación actual, prioridades de políticas y buenas prácticas. En: XIII Conferencia Interamericana de Ministros de Trabajo de la OEA, 39p. 
Olaz Capitán, Ángel José y Ortiz García, Pilar (2017). Aproximación a la caracterización del emprendimiento femenino: una investigación cualitativa en clave competencial. BARATARIA. Revista Castellano-Manchega de Ciencias Sociales, (22), 5I-66. [Fecha de Consulta II de Marzo de 202I]. ISSN: 1575-0825. Disponible en: https://www.redalyc.org/articulo.oa?id=322I/322I 53762003

Organización Internacional del Trabajo. (2019). Panorama Laboral 2019. América Latina y El Caribe (Primera ed., Vol. I) [Libro electrónico]. Oficina Regional para América Latina y el Caribe. https:/www.ilo.org/wcmsp5/groups/public/---americas/---rolima/documents/publication/wcms_732198.pdf

Ramos Soto, Ana Luz (20I5). Sector informal, economía informal e informalidad. RIDE Revista Iberoamericana para la Investigación y el Desarrollo Educativo, 6 (II),. [Fecha de Consulta 10 de Marzo de 202I]. ISSN:. Disponible en: https://www.redalyc.org/articulo.oa?id=498I/498I 503 I 900 I

Ravier, A. (2009). Richard Cantillón y el primer tratado de economía política. Revista de Análisis Institucional Fundación Friedrich A. von Hayek., 3, 97.

Samaniego, Norma (2008). El crecimiento explosivo de la economía informal. Economíaunam, 5 (I3), 30-4I. [Fecha de Consulta 10 de Marzo de 202I]. ISSN: 1665-952X. Disponible en: https://www.redalyc.org/articulo.oa?id=3635/363542894005

Saldarriaga Díaz, Juan Miguel y Vélez-Zapata, Claudia y Betancur Ramírez, Gabriel (2016). Estrategias de mercadeo de los vendedores ambulantes. Semestre Económico, 19 (39), I55-I7I. [Fecha de Consulta II de Marzo de 202I]. ISSN: 0I20-6346. Disponible en: https://www.redalyc.org/articulo.oa?id=I650//6504667/ 007

Trejo, J. (2004). La ocupación en el sector informal 1995-2003. México: INEGI.

Ramos-Soto, A. (2007). Distribución del Ingreso y Sector Informal en las Principales Ciudades del Estado de Oaxaca. Oaxaca: UABJO.

Schneider, F. y Klinglmair, R. (2004). Shadow economies around the world: What do we know? IZA-Discussion Paper 1043, (Marzo).

Schumpeter, J. (1934). Theory of economic development. Cambridge, Mass: Harvard University Press.

Véliz Torresano, José y Díaz Christiansen, Suleen (2014). El fenómeno de la informalidad y su contribución al crecimiento económico: el caso de la ciudad de Guayaquil. Revista de Economía, 